\title{
Stellar formation in Brightest Cluster Galaxies
}

\author{
Tatiana Zapata Pichinao and Gastão B. Lima Neto \\ Instituto de Astronomia, Geofísica e Ciências Atmosféricas, IAG/USP, Brazil \\ email: taz@astro.iag.usp.br - gastao@astro.iag.usp.br
}

\begin{abstract}
We study the interplay between stellar population of the Brightest Cluster Galaxies (BCG) and cluster global properties. We use X-ray properties from Chandra, ROSAT and ASCA observations and BCGs spectra from SDSS-DR6. Using STARLIGHT we determined the star formation history of the BCGs and look for relations with the intra-cluster gas properties. Our first results show no correlation between the recent star formation with x-ray temperature and dynamic state. An important star formation is happening in cool-core Cluster. This new stellar population has low metallicity, pointing to an external source of cold gas to form stars.
\end{abstract}

Keywords. Galaxies: BCGs, spectral synthesis - Galaxy Clusters: cooling flow

\section{Introduction}

Previous studies e.g. have found evidence for recent star formation in the central galaxies of cool-core clusters, but there is an uncertainty about the relation with its environment or the origin of the cold gas. Our aims are to quantify the recent star formation in BCGs and to study how this is linked to global cluster properties.

\section{The Sample and Method}

Our sample is drawn from three X-ray galaxy clusters catalogs: HIFLUGCS (Reiprich \& Böhringer, 2002), RASS-SDSS Galaxy Clusters Survey (Popesso et al., 2004) and Maughan et al. (2008). We selected 90 clusters and groups in the area covered by the Sloan Digital Sky Survey DR6 where the BCG is a dominant galaxy having spectral observations. The redshift interval is $0.006<z<0.5$. We excluded 25 AGNs from our sample based on the BPT diagram (Baldwin, Phillips \& Terlevich, 1981).

To obtain the BCGs star formation history, we use the public spectral synthesis code, STARLIGHT (Cid Fernandes et al. 2005). The base used has 150 SSPs, with six metallicities and 25 different ages. We use the newer MILES library (Sánchez-Blázquez et al., 2006), because it corrects some pathologies obtained with STELIB in passive/elliptical galaxies (Cid Fernandes et al. 2009).

\section{Results}

We focus the discussion in the recent star formation, then we define the young stellar population (YSP) as stars with age $\leqslant 5 \times 10^{7} \mathrm{yr}$.

a) X-ray Temperature: We use gas temperature as a proxy of the cluster mass, then we use the hotter component (Th) of two-temperature fit, accepting a small and cold component in central region of cooling core clusters. (Fig. 1, left) 

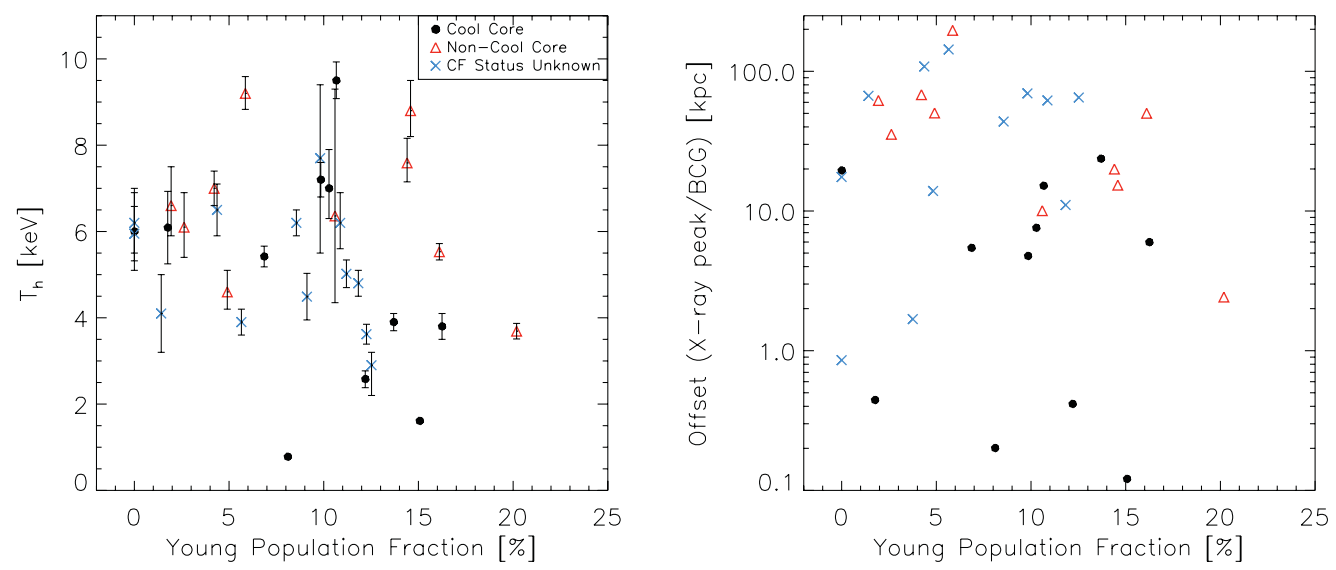

Figure 1. X-ray temperature (left) and distance between x-ray peak and BCG (right) as function of the YSP total luminosity fraction. We found no correlation, but we see that most of the cool-core clusters have a high percent of YSP.
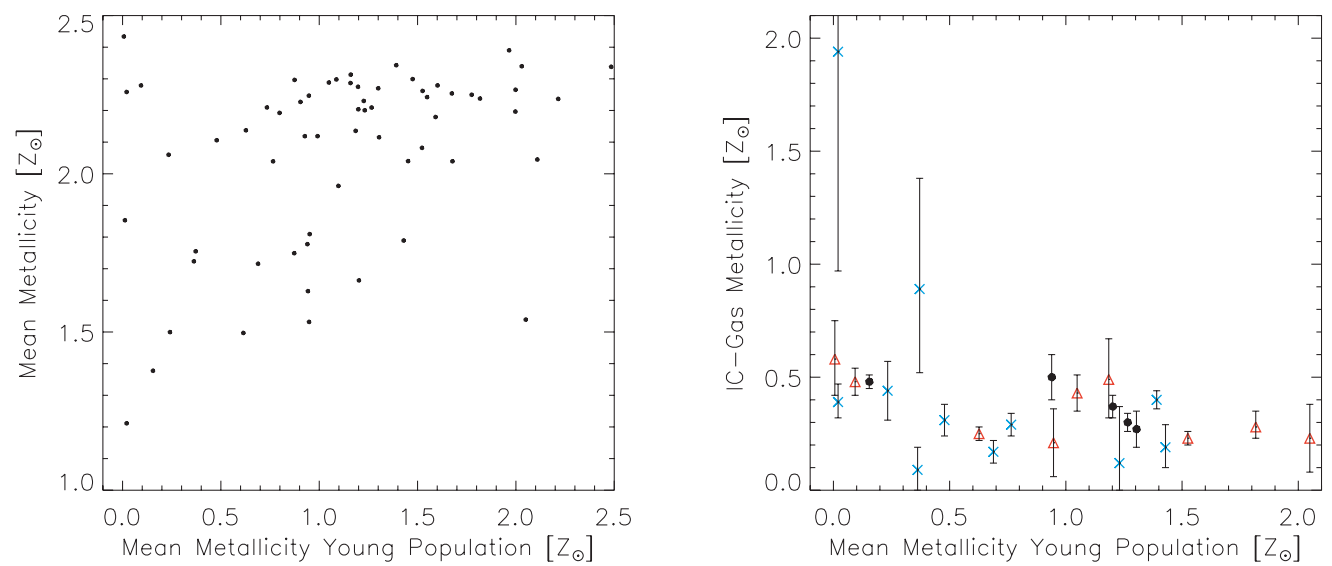

Figure 2. Mean stellar metallicity (left) and intra-cluster gas metallicity (right) as function of mean young stellar metallicity. The lower young stellar metallicity, in respect to all stellar metallicity, points to an external source of cold gas to form stars, but it is not entirely from cooling flow gas as we can infer from the right panel.

b) X-ray peak/BCG Distance: We use $\Omega_{m}=0.25$ and $\Omega_{\Lambda}=0.75$ to calculate distances (X-ray peak position from CHANDRA observations, Fig. 1, right).

c) Metallicity: Flux-weighted mean metallicity.

\section{References}

Baldwin, J. A., Phillips, M. M., \& Terlevich, R. 1981, PASP, 93, 5

Cid Fernandes, R. et al. 2005, MNRAS, 358, 363

Cid Fernandes, R. et al. 2009, RMxAC, 35, 127

Egami, E. et al. 2006b ApJ, 647, 922

Maughan, B. J., Jones, C., Forman, W., \& Van Speybroeck, L. 2003, ApJ, 174, 117

Popesso, P. et al., 2004 A\&A A, 423, 449

Reiprich, T. H. \& Böhringer, H. 2002, ApJ, 567, 716

Sánchez-Blázquez, P. et al. 2006, MNRAS, 371, 703 